\title{
Brain Response to Aversive Taste for Investigating Taste Preference
}

\author{
Chenghong $\mathrm{Hu}^{1}$, Yoshitada Katagiri², Yoshiko Kato ${ }^{3}$, Zhiwei Luo ${ }^{1}$ \\ ${ }^{1}$ Graduate School of System Informatics, Kobe University, Hyogo, Japan \\ ${ }^{2}$ Center for Information and Neural Networks, National Institute of Information and Communications Technology, \\ Osaka, Japan \\ ${ }^{3}$ Graduate School of Human Development and Environment, Kobe University, \\ Hyogo, Japan \\ Email: hchkobe@yahoo.co.jp
}

Received November 24, 2013; revised December 25, 2013; accepted January 12, 2014

Copyright (c) 2014 Chenghong Hu et al. This is an open access article distributed under the Creative Commons Attribution License, which permits unrestricted use, distribution, and reproduction in any medium, provided the original work is properly cited. In accordance of the Creative Commons Attribution License all Copyrights (C) 2014 are reserved for SCIRP and the owner of the intellectual property Chenghong Hu et al. All Copyright (C) 2014 are guarded by law and by SCIRP as a guardian.

\section{ABSTRACT}

To clarify the intrinsic food preference mechanism, we investigated brain neurophysiological responses to unpleasant gustatory stimuli using electroencephalogram (EEG) and near-infrared hemoencepalogram (NIR-HEG) simultaneously. A conventional delayed response task based on Go/Nogo paradigm was adopted to extract real brain response components from spontaneous background signals. We found excessive evoked EEG potential responses to both bitter and sour stimuli, while we didn't find excessive changes in purified water condition. These potentials appeared before $P 3$, hence, they potentially predicted unconscious attention to the gustatory stimuli. We also identified a late contingent negative variation (CNV) and corresponding P3 for sour stimulus. In addition, NIR-HEG responses showed relative changes for every stimulus and we considered that these NIR-HEG signal changes were attributed to the prefrontal cortex activity for regulating negative emotional valence against aversive tastes typically including sour and bitter. In spite of limitation to timing accuracy of taste presentations, the early markers found in this study could be fundamentals for investigating individual food preference.

\section{KEYWORDS}

Electroencephalogram (EEG); Near-Infrared Hemoencepalogram (NIR-HEG); Evoked Potential; Bitter and Sour Taste; Taste Preference

\section{Introduction}

Event-related potentials (ERPs) are a measure of neural activity (derived from the electroencephalogram (EEG), recorded from scalp electrodes) that can be recorded noninvasively from humans while they perform cognitive tasks [1]. The first successfully recorded ERP for taste, one of our basic senses, was published nearly half a century ago [2]. Since then, numerous studies have been concerned with the cognitive processes related to gustatory system. Some have examined the effects of glucose ingestion on ERP and found that glucose ingestion acutely influenced ERP components [3,4]. Moreover, Riby et al. (2008) studied neurocognitive correlates of glucose effects using ERPs and showed that the P3b component had a smaller amplitude, shorter latency and duration when participants were in the glucose condition [4]. So far, many researchers have done a lot of work about gustatory sensation, and some knowledge of taste cognitive processing has been accumulated. However, early sensory ERP deflections to taste have been rarely described and the results are also on disputed [2]. To our knowledge, especially, relatively little is known about the neural mechanisms for taste preference. The primary aim of the current study was to expend the previous research by examining the effect of the aversive taste on cognitive performance to get further understanding about the intrinsic food preference mechanism. In this study, critic acid and caffeine severed as aversive tastants. One important consideration for the utility of critic acid and 
caffeine as stimuli is the role they play in cognitive facilitation. Caffeine is one of the most widely used stimulants worldwide and is generally thought to provide benefits such as enhanced mental alertness, energy and a sense of well-being [5], while the citric acid exists in a variety of fruits and vegetables and it is a key player in our body's ability to create energy [6]. Furthermore, according to a study in the Journal of Clinical Biochemistry and Nutrition, these two aversive tastes may reduce physical fatigue and lessen physiological stress. Considering their importance, although they make people feel uncomfortable or unpleasant, it is significant to investigate the cognitive processes related to aversive taste. We hope the finding in this study will be effective for investigating the children's dietary bias.

We investigated neurophysiological responses to unpleasant gustatory stimuli based on S1-S2 contingent negative variation (CNV) paradigm, to clarify the intrinsic food preference mechanism. The CNV was one of the first ERP components to be described and it is a slow negative-going ERP elicited by a warning stimulus that requires anticipation of a target stimulus $[7,8]$. After discovery of the CNV, Loveless and Sanford (1975) and Weerts and Lang (1973) suggested that the component is quantifiable into two distinct subcomponents: an "early" CNV and a "late" CNV $[9,10]$. The presence of the early CNV is generally thought to be a cortical reflection of controlled, rather than automatic, psychological processes in response to an S1 that requires anticipation of a subsequent S2 [7,11], while the late CNV is measured just prior to the onset of the target stimulus, and reflects the additional contribution of cortical resources required for motor response preparation [12,13]. To date, there are many researches which describe what stimulus characteristics can affect the characteristics of the CNV. For example, attention and expectancy, intensity, modality, duration, stimulus rate, probability, stimulus relevance and pitch discrimination can affect the CNV component [14, 15]. However, there also exist some debates regarding which cognitive processing the CNV is associated with. Considerable evidence suggested that the early component of the CNV of the ERP, thought to reflect anticipated cognitive effort, is sensitive to the effortful process that contributes to the manifestation of prejudice [16]. Since several patterns of cognitive processes are measurable by the early, late CNV may be related to the expression of the prejudice. In the present study, we expected that the S1-S2 CNV paradigm would be adapted to quantify the cognitive processing during taste processing for investigating neural mechanisms of taste preference.

Near-infrared hemoencephalogram (NIR-HEG) is a relatively new HEG neurofeedback system. It covers the human prefrontal lobe and can measure changes in the local oxygenation level of the blood. By far, this device is the simplest neurofeedback system available, which provides you succinct, real-time feedback of your brain function. In addition, in macaque monkeys, the cortical processing of gustatory information takes place in the insula/operculum $[17,18]$. Correspondingly, the orbitofrontal cortex (OFC) receives projections from the insula/ operculum and has been associated with the reward value of tastes [19]. Furthermore, similar results have been found in human, and increasing researches suggested that the lateral prefrontal cortex (LPFC) is closely related to taste and other food-related activities [20,21]. The importance of LPFC in taste processing is gradually drawing attention [20]. To date, some studies examined LPFC functions with taste and indicated that the LPFC is involved in the taste processing. However, to our knowledge, only preliminary steps have been taken to the direct investigation of the underlying neural mechanisms of taste, especially for food preference.

\section{Materials and Methods}

\subsection{Participants}

5 right handed volunteers participated in the experiments (three female and two male). They were between 25 and 50 years of age. Prior to the recording session, all participants completed a health questionnaire and none of them had any past or current psychiatric and neurological diseases and self-reported taste disturbance. Participants were instructed to fast for two hours but drink normally before running experiment. This study was approved by the Human Ethics Committee of Health Promotion and Education Graduate School of Human Development and Environment in Kobe University. Subjects gave written informed consent prior to the experiment.

\subsection{Stimuli}

In present study, we selected the sour solution (1\% citricacid) and bitter solution (0.01\% caffeine) as tastants. The choice of these solutions was based on our previous experiments where stimuli with different concentrations had been employed. Meanwhile, purified water was selected as a control condition to minimize the artificial noise from somatosensory effects, swallowing effects and other brain function.

\subsection{Experimental Procedure and Data Acquisition}

Before the testing session, each subject received detailed instructions regarding the experiment. They were instructed to minimize body and brain movement as possible and asked to remain relaxed throughout the experiment. In order to ensure subjects understood the task requirements, pre-experiments were given to practice.

During the EEG and NIR-HEG recording session, the 
subjects were seated in a comfortable chair in a quiet room (Figure 1). A conventional delayed response task paradigm was adopted for detecting neurological signs responding to gustatory stimuli. Figure 1 showed the experimental protocol as one trail. S1 is a warning signal using a click, and S2 seconds later we used another click as imperative signal to order the experimenter inject the stimulus. The subject tastes the stimulus for 3 seconds. Stimulus was manually injected into the subject's mouth via a hand-held syringe connected to a tube in quantities of $0.2 \mathrm{ml}$ each time. Each stimulus was presented 30 times repeatedly and continuously.

The ERPs were recorded using NeXus-32 and BioTrace + Software. Electrodes were placed at standard positions according to the International 10 - 20 EEG system, while the NIR-HEG covered the prefrontal lobe. In each condition (purified water, sour solution and bitter solution), the EEG was recorded for $2.5 \mathrm{~min}$, and signs

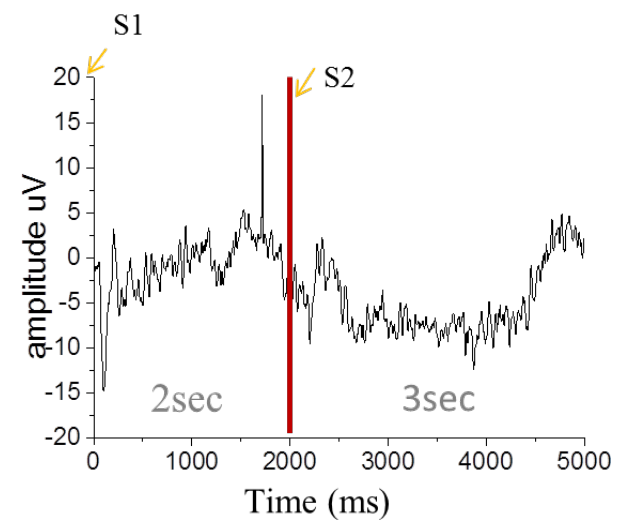

(a)

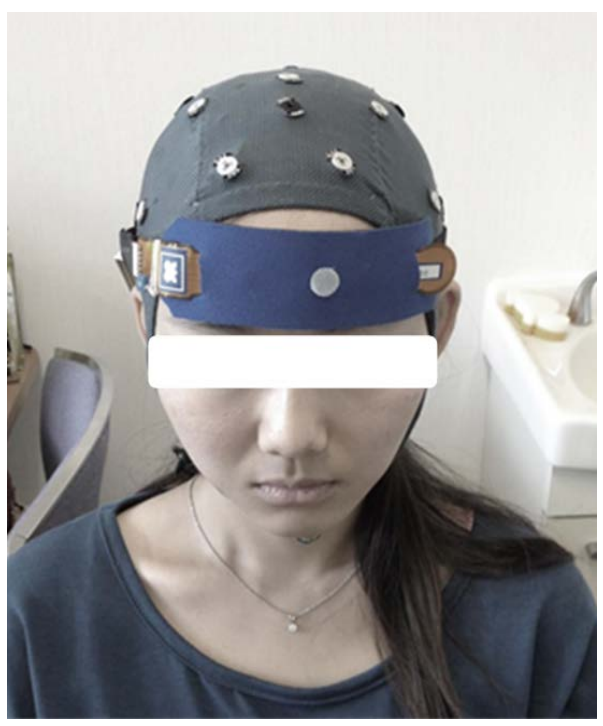

(b)

Figure 1. The experimental protocol and instruments. (a) One trail of the experimental protocol. (b) Experimental conditions of EEG and NIR-HEG. (5s) were extracted as an average value of 30 times. Data was filtered offline between $0.05 \mathrm{~Hz}$ and $45 \mathrm{~Hz}$. The intensity of the stimulus was rated by the subjects as a subjective evaluation on the dislike-like scale after the EEG session.

\section{Result}

\subsection{EEG Data}

Previous research indicated that the P3 component is centered on the parietal and central scalp regions while the CNV appears most prominently at the vertex and is bilaterally symmetrical [15]. Therefore, our analysis focused on the $\mathrm{Cz}$ electrodes. Conversely, the ERP components have been shown to be centralized on the central scalp. Figure 2 summarized the ERP data response to taste stimuli for the $\mathrm{Cz}$ position. After averaging the gustatory ERPs recorded, two positive peaks (P1, P2) and two negative peaks (N1, N2) were observed in the bitter and sour condition. To confirm these gustatory ERPs were truly evoked by bitterness and sourness, we also examined the potentials evoked by purified water and compared it with the components evoked by citric acid and caffeine. These peaks, P1, P2, N1, N2, were not found in the purified water condition obviously. The amplitudes of these responses ranged between 5 and $15 \mu \mathrm{V}$, and the sour taste elicited responses of greater amplitude than bitterness. In present study, we also identified a $\mathrm{CNV}$ component in a late stage for the sour and corresponding clear P3 (Figure 2(c)).

\subsection{NIR-HEG Data}

Meanwhile, in present study, we had a try to use NIRHEG to monitor the activation of human prefrontal cortex, which plays an important role in human higher cognitive processing. The individual signal changes in hemoglobin during tasting bitter and sour solutions were exhibited in Figures 3 and 4. Figure 3 showed the responses to bitter stimulus for two subjects. Although they showed different responses individually, in general, both of them showed a relative decrease of hemoglobin during tasting bitter solution. On the other hand, there was a relative increase in sour solution processing exhibited in Figure 4. In addition, the responses of sourness from NIR-HEG also showed more sensitive than those induced by bitterness. For statistical analysis, significances between purified water, bitter and sour solutions respectively could be found in present study.

\section{Discussion}

The primary aim of the current study was to expend the previous research by examining the effect of the aversive taste on cognitive performance for investigating taste 

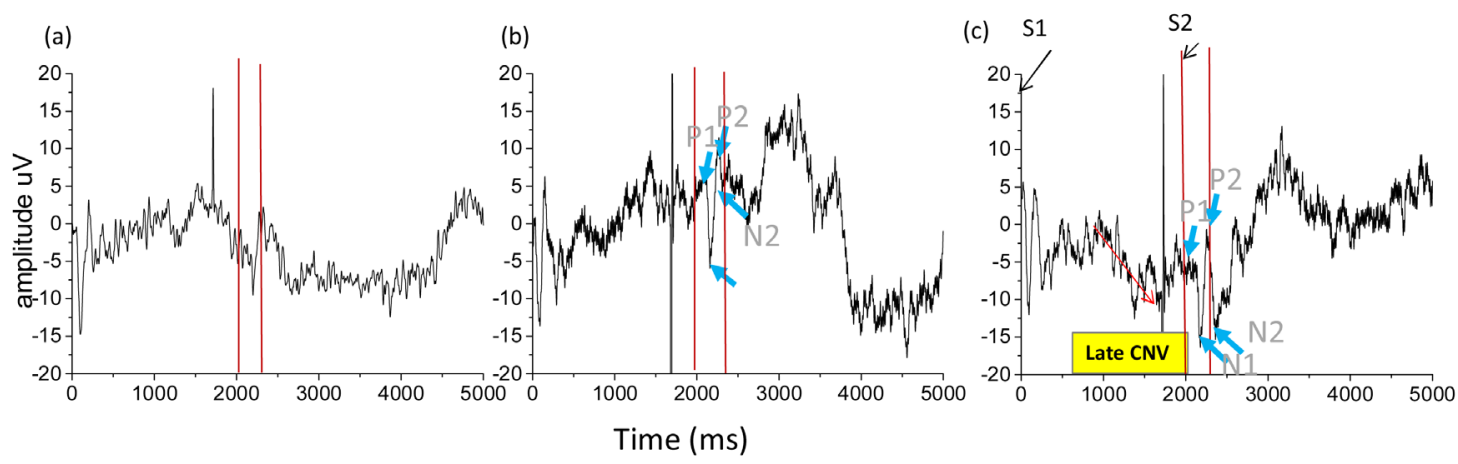

Figure 2. ERPs to taste stimuli. (a) Represents the evoked potential response to water, (b) For bitter taste, and (C) For sour taste.

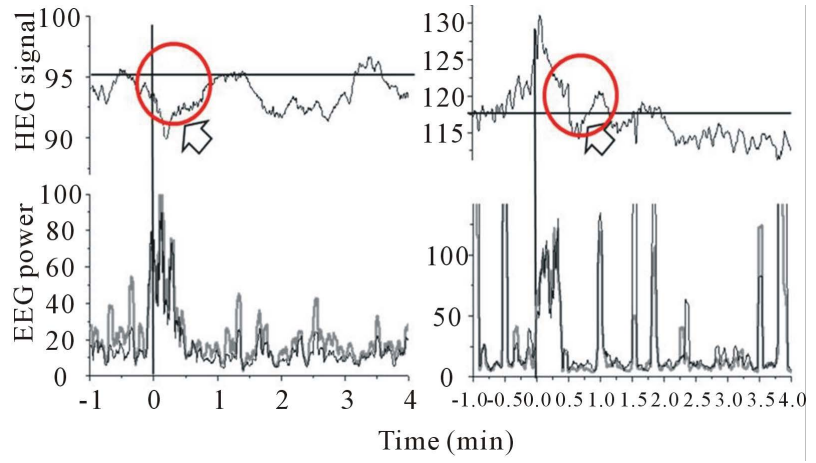

Figure 3. HEG and EEG responses to bitter stimuli (Experiments were performed with two participants).

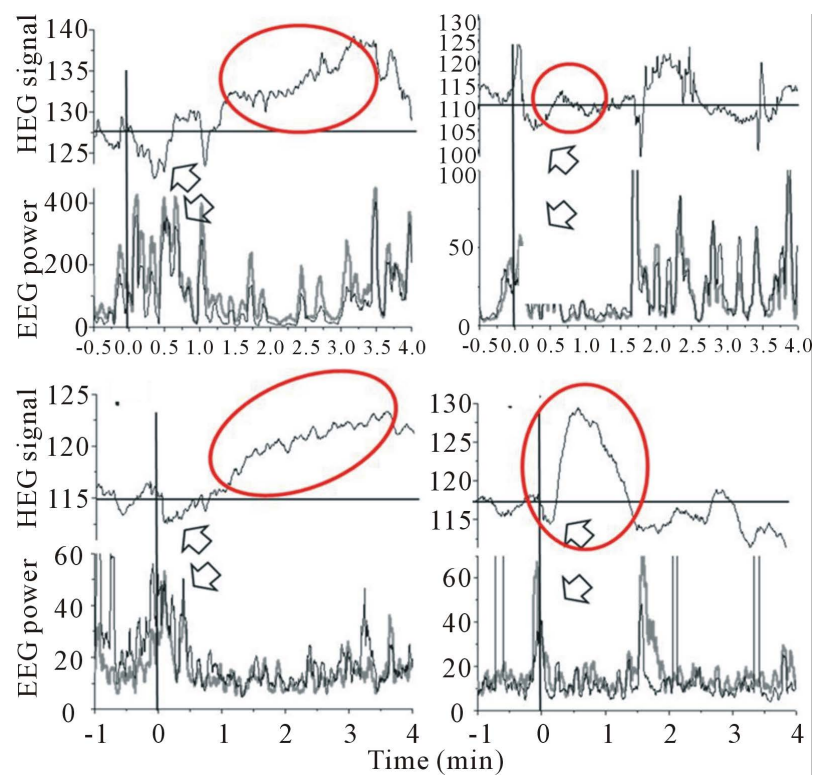

Figure 4. HEG and EEG responses to sour stimulus. (Experiments were performed with four participants).

preference. The results showed some electrophysiological early markers appeared during tasting citric acid and caffeine, and we suggested that these components we found would be only attributed to sour and bitter tastes. As these potentials appeared before P3, we considered they would be unconscious electrophysiological early markers for attention. The early sensory ERP deflections to taste found in present study were in agreement with previous researches, which reported P1, P2 and N2 were observed for salt, glucose and electric taste [20-24]. In addition, this study showed the sour taste gave larger rise than bitter taste. It may be due to the quality and intensity/concentration of stimulus, which have been suggested to affect amplitude increase of the evoked potential [24].

In this study, the effects of aversive taste on the CNV wave were assessed in order to explore the utility of the $\mathrm{CNV}$ as a marker of activity of taste preference. In line with the expectations, we identified CNV in a late stage for the sour and corresponding clear P3 (Figure 2(c)). Between S1 and S2, two waves can be observed, the early and the late CNV wave $[9,10]$. In this study, the late CNV wave was investigated in sour solution condition. The late CNV is measured just prior to the onset of the target stimulus, and reflects the additional contribution of cortical resources required for motor response preparation $[16,25]$. Especially, relevant to present study, the late $\mathrm{CNV}$ was suggested to be related to the prejudice for sour taste, which matched the subjects' self-reported that sourness made them feel more unpleasant.

For the second part of this study, we used NIR-HEG to monitor the human prefrontal cortex activity during tasting aversive tastes. Previous studies reported taste reward areas are located in different OFC region. For example, Roll et al. (1988) studies on the responsiveness of neurons in the fontal operculum gustatory cortex and found the firing rates of neurons in OFC are modulated by hunger and satiety. It suggested the OFC region represent the gustatory reward value [18]. O’Doherty et al. (2001), using fMRI, showed the individual differences for areas activated glucose and salt and the group analysis indicated the inferior medial OFC responds to changes in pleasantness associated with eating [26]. There are also another studies researching on the LPFC function with taste based on different task and design. Anyway, these physiological studies have focused on a variety of issues, but all of them reported that the OFC contains a signifi- 
cant cortical taste projection area. We believed the PFC plays a critical role in modulating taste information processing, and the experiment here indicated the prefrontal cortex was involved in sour and bitter taste cognitive processing, which was consistent with previous findings. In future work, we will do more investigation to get more specific information of human prefrontal work about gustatory system.

In present study, we used sour solution (1\% citric acid) and bitter solution ( $0.01 \%$ caffeine) as gustatory stimuli. Future work will be needed to examine whether the amplitudes and latencies of the gustatory ERPs peaks are dependent on the taste qualities (such as sweet, salt and umami) or the concentration. In addition, it must be admitted that our study was performed on 5 participants and there is a possibility that the ERPs amplitude is under the level of statistical significance. We will extend our experiments among more subjects to explore this possibility in the future.

\section{Conclusion}

Taken together, we reported an EEG study to investigate taste preference using aversive stimuli. The early markers before P3 were related to sour and bitter taste and we considered they would be unconscious about the electrophysiological early markers for attention. Especially, the CNV appeared to be a biomarker for taste preference. Furthermore, this study gave further evidence which supported that the prefrontal cortex was involved in taste cognitive processes using NIR-HEG. Since this study was a pilot, we would extend such investigation to further more participants having various food preferences.

\section{REFERENCES}

[1] T. R. Bashore and M. van der Molen, "Discovery of P300: A Tribute,” BiolPsychology, Vol. 32, No. 2-3, 1991, pp. 467-475. http://dx.doi.org/10.1016/0301-0511(91)90007-4

[2] K. Ohla, N. A. Busch and J. N. Lundström, "Time for Taste: A Review of the Early Cerebral Processing of Gustatory Perception," Chemosencs Percept, Vol. 5, No. 1, 2012, pp. 87-99. http://dx.doi.org/10.1007/s12078-011-9106-4

[3] M. A. Smith, L. M. Riby, S. I. Sunram-Lea, J. A. van Eekelen and J. K. Foster, "Glucose Modulates Event-Related Potential Components of Recollection and Familiarity in Healthy Adolescents," Psychopharmacology (Berlin), Vol. 205, No. 1, 2009, pp. 11-20. http://dx.doi.org/10.1007/s00213-009-1509-4

[4] L. M. Riby, J. McLaughlin and D. M. Riby, "Lifestyle, Glucose Regulation and the Cognitive Effects of Glucose Load in Middle-Aged Adults,” British Journal of Nutrition, Vol. 100, No. 5, 2008, pp. 1128-1134. http://dx.doi.org/10.1017/S0007114508971324

[5] F. Koppelstaetter, T. D. Poeppel, C. M. Siedentopf, et al.,
"Does Caffeine Modulate Verbal Working Memory Processes? An fMRI Study,” Neuroimage, Vol. 39, No. 1, 2008, pp. 492-499.

http://dx.doi.org/10.1016/j.neuroimage.2007.08.037

[6] S. Kozawa and Y. Fukuda, "Standard Physiology,” Igakushoin, Tokyo, 2009, p. 294.

[7] T. Picton and S. Hillyard, "Endogenous Component of the Event-Related Brain Potential,” In: T. Picton, Ed., Human Event-Related Potentials: EEG Handbook, Elsevier, Amsterdam, 1988, pp. 361-426.

[8] W. Walter, R. Cooper, V. Aldridge, W. McCallum and A. Winter, "Contingent Negative Variation: An Electric Sign of Sensori-Motor Association and Expectancy in the Human Brain,” Nature, Vol. 203, No. 4943, 1964, pp. 380384. http://dx.doi.org/10.1038/203380a0

[9] N. E. Loveless and A. J. Sanford, "The Impact of Warning Signal Intensity on Reaction Time and Components of the Contingent Negative Variation,” Biological Psychology, Vol. 2, No. 3, 1975, pp. 217-226.

http://dx.doi.org/10.1016/0301-0511(75)90021-6

[10] T. C. Weerts and P. J. Lang, "The Effects of Eye Fixation and Stimulus and Response Location on the Contingent Negative Variation (CNV),” Biological Psychology, Vol. 1, No. 1, 1973, pp. 1-19. http://dx.doi.org/10.1016/0301-0511(73)90010-0

[11] R. Shiffrin and W. Schneider, "Controlled and Automatic Human Information Processing: II. Perceptual Learning, Automatic Attending, and a General Theory,” Psychological Review, Vol. 84, No. 2, 1977, pp. 127-190. http://dx.doi.org/10.1037/0033-295X.84.2.127

[12] E. Damen and C. Brunia, "Is a Stimulus Conveying TaskRelevant Information a Sufficient Condition to Elicit a Stimulus-Preceding Negativity," Psychophysiology, Vol. 31, No. 2, 1994, pp. 129-139. http://dx.doi.org/10.1111/j.1469-8986.1994.tb01033.x

[13] C. Brunia and E. Damen, "Distribution of Slow-Potentials Related to Motor Preparation and Stimulus Anticipation in a Time Estimation Task," Electroencephalagraphy and Clinical Neurophysiology, Vol. 69, 1988, pp. 234-243.

[14] B. G. Frost, R. A. Neill and B. Fenelon, “The Determinants of the Non-Motoric CNV in a Complex, Variable Foreperiod, Information Processing Paradigm,” Biological Psychology, Vol. 27, No. 1, 1988, pp. 1-21. http://dx.doi.org/10.1016/0301-0511(88)90002-6

[15] J. J. Tecce, "Contingent Negative Variation (CNV) and Psychological Processes in Man,” Psychological Bulletin, Vol. 77, No. 2, 1972, pp. 73-108. http://dx.doi.org/10.1037/h0032177

[16] P. Chiu, N. Ambaby and P. Deldin, “Contingent Negative Variation to Emotional in- and Out-Group Stimuli Differentiated High- and Low-Prejudiced Individuals,” Journal of Cognitive Neuroscience, Vol. 16, No. 10, 2004, pp. 1830-1839. http://dx.doi.org/10.1162/0898929042947946

[17] E. T. Rolls and T. R. Scott, "Central Taste Anatomy and Neurophysiology,” In: R. K. L. Doty, Ed., Handbook of Olfaction and Gustation, 2nd Edition, Marcel Dekker, Inc., New York, 2003, pp. 679-705.

[18] E. T. Rolls, T. R. Scott, Z. J. Sienkiewicz and S. Yaxley, 
“The Responsiveness o Fneurons in the Fontal Operculum Gustatoryd Cortex of the Macaque Monkeys Is Independent of Hunger," The Journal of Physiology, Vol. 397, 1988, pp. 1-12.

[19] E. T. Rolls, Z. J. Sienkiewicz and S. Yaxley, "Hunger Modulates the Responses Togustatory Stimuli of Single Neurons in the Caudolateral Orbitofrontal Cortex of the Macaque Monkey," European Journal of Neuroscience, Vol. 1, No. 1, 1989, pp. 53-60. http://dx.doi.org/10.1111/j.1460-9568.1989.tb00774.x

[20] M. L. Kringebach, I. E. de Araujo and E. T. Rolls, “TasteRelated Activity in the Human Dorsolater Prefrontal Cortex,” Neuroimage, Vol. 23, No. 4, 2004, pp. 781-788. http://dx.doi.org/10.1016/j.neuroimage.2003.09.063

[21] M. Okamoto, H. Dan, A. K. Singh, F. Hayakawa, V. Jurcak, T. Suzuki, K. Kohyama and I. Dan, "Prefrontal Activity during Flavor Difference Test: Application of Functional Near-Infrared Spectroscopy to Sensory Evaluation Studies,” Appetite, Vol. 47, No. 2, 2006, pp. 220-232. http://dx.doi.org/10.1016/j.appet.2006.04.003

[22] C. Mizoguchi, T. Kobayakawa, S. Saito and H. Ogawa,
"Gustatory Evoked Cortical Activity in Humans Studied by Simultaneous EEG and MEG Recording," Chemical Senses, Vol. 27, No. 7, 2002, pp. 629-634. http://dx.doi.org/10.1093/chemse/27.7.629

[23] M. Wada, "Evoked Responses to Taste Stimulation,” The International Tinnitus Journal, Vol. 11, No. 1, 2005, pp. 43-47.

[24] K. Ohla, U. Toepel, J. le Coutre and J. Hudry, "Electrical Neuroimaging Reveals Intensity-Dependent Activation of Human Cortical Gustatory and Somatosensory Areas by Electric Taste,” Biological Psychology, Vol. 85, No. 3, 2010, pp. 446-455.

[25] C. Brunia and E. Damen, "Distribution of Slow-Potentials Related to Motor Preparation and Stimulus Anticipation in a Time Estimation Task," Electroencephalagraphy and Clinical Neurophysiology, Vol. 69, 1988, pp. 234-243.

[26] J. O’Doherty, E. T. Rolls, S. Francis, R. Rowtel and F. McGlone, "Representation of Pleasant and Aversive Taste in the Human Brain,” Journal of Neurophysiology, Vol. 85, No. 3, 2001, pp. 1315-1321. 